\title{
A Discrete Choice Experiment to Derive Health Utilities for Aromatic L-Amino Acid Decarboxylase (AADC) Deficiency in France
}

\author{
Adam B Smith ${ }^{\prime}$ \\ Andria Hanbury $\mathbb{( D}^{\prime}$ \\ Jennifer A Whitty $\mathbb{D}^{2}$ \\ Igor Beitia Ortiz de Zarate ${ }^{3}$ \\ Florence Hammes ${ }^{3}$ \\ Gérard de Pouvourville ${ }^{4}$ \\ Katharina Buesch ${ }^{5}$ \\ 'York Health Economics Consortium, \\ University of York, York, UK; ${ }^{2}$ Norwich \\ Medical School, University of East Anglia, \\ Norwich, UK; ${ }^{3}$ PTC Therapeutics, Paris, \\ France; ${ }^{4}$ Department of Economics, \\ ESSEC, Paris, France; ${ }^{5}$ PTC Therapeutics, \\ Zug, 6300, Switzerland
}

Purpose: Cost-effectiveness evaluations of interventions require health utility data. However, in medical conditions, such as aromatic L-amino acid decarboxylase (AADC) deficiency, this presents problems due to the rarity of the disease. The study aim therefore was to employ a discrete choice experiment (DCE) to generate health utilities for AADC deficiency.

Methods: A previous literature review, clinician and parent interviews had identified six key AADC deficiency attributes: mobility, muscle weakness, oculogyric crises (OCG), feeding ability, cognitive impairment and screaming. A representative sample of the French general population was recruited. Participants rated 5 health state vignettes describing AADC deficiency using time-trade-off (TTO) and standard gamble (SG). Additionally, participants rated the worst health state using the Health Utility Index version 3 (HUI3). Subsequently, participants completed DCE 11 choice sets. Indirect DCE part-worth utilities were converted to health utilities using the anchors from the TTO, SG and HUI3.

Results: The DCE was completed online by 1001 participants (50.9\% female; mean age 45.7 years). Most participants $(596,59.5 \%)$ provided consistent responses to the repeated choice task. Five models were evaluated, and one preference reversal ("head control"/"sitting unaided") was identified in all models. The rescaled utilities ranged from 0.3891 to 0.5577 (difference of 0.17 utilities) for TTO anchors corresponding to the worst (633233) and best (111111) health states. Health utilities ranged from 0.5534 to 0.7093 for the SG anchors. The disutility associated with a transition from "no problems walking" to "bedridden" was -0.0533 , whereas disutility of moving from "constant screaming" relative to "no screaming" was -0.0248 . The disutility associated with daily OCG was -0.0167 . Disutilities for the other attributes were small although there were exceptions.

Conclusion: A DCE was used to derive health utilities for AADC deficiency. These health utilities will subsequently be used in an economic model evaluating an AADC deficiency intervention.

Keywords: aromatic L-amino acid decarboxylase deficiency, discrete choice experiment, health state utilities, health utilities

\section{Introduction}

Health-related quality of life assessments (HRQoL) are crucial to understanding the impact of medical conditions and, in the form of health utilities, are critical for the process of evaluating the cost-effectiveness of interventions. However, in rare diseases, especially those involving paediatric populations, measuring HRQoL is problematical given the
York Health Economics Consortium,

Enterprise House, Innovation Way,

University of York, York, YOI0 5NQ, UK

Tel +44 1904323620

Fax +441904323628

Email adam.smith@York.ac.uk 
nature of the condition and patients affected. Aromatic L-amino acid decarboxylase (AADC) deficiency is a rare, genetic condition typically presenting in infancy or early childhood with only around 150 reported cases worldwide. ${ }^{1}$ Common symptoms include hypotonia, developmental delay, movement disorders, and oculogyric crises. ${ }^{2}$ Deriving health utilities for AADC deficiency using instruments such as the EuroQol 5D-5L (EQ-5D-5L) ${ }^{3}$ - the stated preferred instrument for health-care agencies such as the Haute Autorité de Santé $(\mathrm{HAS})^{4}-$ is not possible. The low incidence of AADC deficiency also means that it is difficult to derive robust health state utilities from parents or caregivers ${ }^{5}$ given that the population size from which participants are drawn would consequently be very small. Therefore, other approaches need to be applied to generate health utilities for AADC deficiency, as have been employed in other rare diseases, such as proxyratings from clinicians. ${ }^{6,7}$ Given that the sample size, ie, number of clinicians in these proxy studies is often small, they may not be sufficient to derive robust utility data. ${ }^{8}$ Other methods such as time trade-off (TTO) and standard gamble tasks (SG) ${ }^{9}$ have been used to derive health state utilities for rare conditions, ${ }^{10,11}$ although these approaches tend to provide global utilities for the health states, rather than utilities or disutilities for individual levels of the key symptoms.

An alternative to generate utilities is discrete choice experiments (DCE) where participants are presented with a number of choice sets comprised of symptoms (attributes) and levels (degrees of severity) for the condition in question. Choice sets consist of two (or more) health state profiles with differing combinations of levels and the participant is asked to select one of these profiles. ${ }^{12}$ This process allows preferences to be derived for the given health states. DCEs have been used in rare diseases previously. ${ }^{13,14}$ Although the parameters derived from DCEs are not health utilities, they can be anchored ${ }^{15}$ to utilities from preference-based methods such as TTO and SG utilities to, for instance, derive quality-adjusted life years (QALYs) for economic models.

The aim of this study was to derive health state utilities for the key attributes of AADC deficiency using a DCE to complement a vignette study in a French sample. The health utilities will be used in an economic model evaluating a novel gene therapy for AADC deficiency.

\section{Participants and Methods}

\section{Participants}

In order to ensure that the sample was as representative of the French population as possible a sample size of 1000 respondents was selected. Basic socio-demographic details (age, biological sex (male/female/prefer not to say), parental status, and region of residence in France), were collected from respondents and used to screen for eligibility. Eligible respondents included France residents, aged $\geq 18$ years. Parents and caregivers of children with life-threatening or life-limiting conditions were screened out of the study in order to reduce any potential bias. The study was conducted online. Participants received a nominal incentive (redeemable points) if they completed the study in full.

The study was submitted for review to the University of York's Health Sciences Research Governance Committee and received ethics approval on 20 March 2020 and conducted in accordance with the Declaration of Helsinki.

\section{DCE Design, Procedure and Analysis} Overview

The discrete choice experiment was part of a larger study that has been described in detail elsewhere incorporating a vignette study in both the United Kingdom (UK) and France and DCE conducted in the UK. ${ }^{11,16-18}$ The vignettes and DCEs were developed in English in the first instance $^{16}$ before translation into French.

\section{DCE}

The DCE attributes were: mobility; muscle weakness or floppiness; oculogyric crises; feeding support; cognitive impairment; and screaming. The attribute levels were as follows: 6 for the "mobility" attribute, two levels were chosen for "feeding support" (unable/able to feed themselves) and three levels were selected for the other attributes (except "feeding support") to reflect mild, moderate and severe symptoms (Table 1).

\section{Sample Size}

Orme's rule of thumb was applied ${ }^{19}$ for the sample size, resulting in a minimum sample size of 167 respondents assuming each respondent completes 9 choice sets, each set contains two alternative health states, and a maximum of 6 levels are used to describe each symptom. ${ }^{20}$

\section{Procedure}

The procedure follows that described in detail in the UK DCE study. ${ }^{20}$ In brief, participants were provided with an introduction to the study. Once informed consent had been provided by the participants some basic sociodemographic details were collected. Participants then completed a series of TTO and SG tasks. ${ }^{11,17}$ As part of this process, participants were shown a vignette describing the 
Table I DCE Symptoms and Levels (and Dummy Code for Analysis)

\begin{tabular}{|c|c|c|}
\hline Symptom & Level & Dummy Code \\
\hline \multirow[t]{6}{*}{ Mobility } & Bedridden & Mob6 \\
\hline & Head control & Mob5 \\
\hline & Able to sit unaided & Mob4 \\
\hline & Able to stand with support & Mob3 \\
\hline & Able to walk with assistance & Mob2 \\
\hline & Able to walk without assistance & MobI (Referent) \\
\hline \multirow[t]{3}{*}{ Muscle weakness/floppiness } & Severe weakness & Weak3 \\
\hline & Moderate weakness & Weak2 \\
\hline & No weakness & WeakI (Referent) \\
\hline \multirow[t]{3}{*}{ Oculogyric crises (OCG) } & Daily OGC & Ocg3 \\
\hline & Occasional OGC & Ocg2 \\
\hline & No OGC & Ogcl (Referent) \\
\hline \multirow[t]{2}{*}{ Feeding support } & Not able to feed themselves & Feed2 \\
\hline & Able to feed themselves & FeedI (Referent) \\
\hline \multirow[t]{3}{*}{ Cognitive impairment } & Severe & $\operatorname{Cog} 3$ \\
\hline & Moderate & $\operatorname{Cog} 2$ \\
\hline & No & CogI (Referent) \\
\hline \multirow[t]{3}{*}{ Screaming } & Constantly & Scrm3 \\
\hline & Frequently & Scrm2 \\
\hline & Not at all & Scrml (Referent) \\
\hline
\end{tabular}

worst health state ("bedridden") (Figure 1) and asked to imagine and then complete the French-language version of Health Utilities Index-3 (HUI3). ${ }^{22-25}$ The HUI3 is a 15item instrument with 8 domains corresponding to: Vision, Hearing, Speech, Ambulation, Dexterity, Emotion, Cognition and Pain. The child-proxy version of the HUI3 was used in this task. Following this, participants completed the DCE.

\section{Experimental Design for the DCE}

The DCE design replicated that utilised in the UK study: ${ }^{18}$ 108 choice sets were created divided into 12 blocks of 9 to create a main effects orthogonal design using NGene statistical design software. ${ }^{21}$ Each respondent completed 10 choice sets (Figure 2): the 9 choice sets (from one of the 12 blocks) along with an additional choice. For the latter, one of the choice sets (the 2nd) in each block had been reversed to evaluate internal consistency. The choice sets were randomised across participants (Figure 3). For each choice set, participants which of two alternative health states they would prefer their child to have with each health state defined by the 6 symptoms described above (Table 1).

\section{Analysis}

\section{Analysis of DCE Data}

NLogit statistical software (version 6) was used for analysis of the DCE data. ${ }^{26}$ The relative importance of the symptom levels in driving health state choice was estimated through multinomial logit (MNL) regression analysis of the choice data. The utility function for the choice model was specified as a linear additive function of the main effects for each symptom.

Only the 9 choice sets included in the statistical design (and not the repeated 10th choice set included as 


\section{Health State Description}

\section{Bedridden (Worst Health State)}

Imagine that you are a parent or caregiver of a child with a severe medical condition. This medical condition means that:

- Your child is bedridden and unable to move by themselves. This means that your child is unable to lift and control their head, crawl, sit, or stand.

- They are unable to feed themselves and may need to be fed through a tube.

- They have very poorly developed muscle tone meaning their body, arms and legs are very floppy. This means your child is unable to grasps or hold onto things.

- Your child will also frequently experience painful muscle spasms, and their arms and legs may move involuntarily with sudden jerking or twisting.

- Your child screams constantly throughout the day and night.

- Your child experiences something called an "oculogyric crisis". This is where their eyes rotate or roll in unusual ways, similar to an epileptic seizure. These may last for up to several hours, several times a day.

- Your child will have problems sleeping.

- Your child will have severe abdominal problems, such as constipation or diarrhoea.

- They will be extremely irritable and agitated.

- Your child is unable to follow objects with their eyes and unable to recognise and interact with people. They can understand simple words but are not able to speak.

- Other symptoms include severely blocked nose ("nasal congestion"), which may lead to serious chest infections, as well as excessive drooling, excessive sweating and extreme tiredness.

Figure I Example of health state vignette.

Please read the symptom groups below carefully, and decide that if your child had these symptoms, which group of symptoms would you prefer they had, group A or group B.

\begin{tabular}{|l|l|l|}
\hline & \multicolumn{1}{|c|}{ Symptom group A } & \multicolumn{1}{c|}{ Symptom group B } \\
\hline Mobility & Able to sit unaided & Head control \\
\hline Muscle weakness / floppiness & No weakness & Severe weakness \\
\hline Oculogyric crises & Occasional OGC & No OGC \\
\hline Feeding support & $\begin{array}{l}\text { Able to feed } \\
\text { themselves }\end{array}$ & $\begin{array}{l}\text { Not able to feed } \\
\text { themselves }\end{array}$ \\
\hline Cognitive impairment & Severe & Moderate \\
\hline Screaming & Not at all & Frequently \\
\hline
\end{tabular}

Symptom group $\mathrm{A}$

$\bigcirc$
Symptom group B

Figure 2 Example choice set.

consistency check) were included in the regression analyses. MNL models were estimated on the complete sample, and on subsamples (eg, excluding participants always choosing the same alternative in every choice set, those always selecting the same alternative or giving an inconsistent answer to the tenth repeated choice set).

\section{Rescaling of Health States to QALY Scale}

The mean utility values for the worst health state (profile score, 633233) derived using the HUI3, and those for the best health state (profile score, 111111) derived from the TTO and SG tasks were used anchor the DCE health state preferences onto a utility scale ( 0 to 1 or dead to perfect health). ${ }^{15}$

\section{Results \\ Sample Characteristics}

There were 1001 respondents who completed the survey and included for the DCE analysis. Their sociodemographic 


\section{Instructions for the DCE}

This part of survey consists of 10 hypothetical scenarios describing 6 symptoms of AADC deficiency. You will be asked to imagine being the main parent or caregiver of a child with AADC deficiency. For each scenario there are two sets of symptoms. You will be asked to make a choice between one of these sets of symptoms. The symptoms are described in more detail below:

- Mobility: This describes whether the child is bedridden, has head control, or is able to sit, stand with support and walk with assistance;

- Muscle weakness / floppiness: This describes the child's muscle strength, as children with AADC deficiency can be very floppy due to weak muscles; this means they are unable to grasp or hold onto things

- Oculogyric crises: This is unusual eye movements where the eyeball rolls upwards or from side and can last for several hours; this is very discomforting for the child

- Feeding difficulties or support: This describes whether or not the child is able to feed themselves;

- Cognitive impairment: This refers to the child being unable to talk or understand words and sentences;

- Screaming / crying: This is a common symptom of AADC deficiency where the child screams for lengthy periods of time.

Figure 3 Description of DCE.

characteristics are presented in Table 2. Just over half (510, $50.9 \%$ ) of respondents were female, the average age of respondents was $45.68 \pm 16.78$ years.

\section{Choice Data}

The 12 blocks were each completed by between $7.6 \%$ and $9.2 \%$ of respondents. A summary of data related to completion of the survey and choices is provided in Table 2. The median survey completion time was 11.7 minutes (interquartile range 8.3 to 17.9 minutes). A minority $10 \%$ completed in less than 399 secs (6.7 mins). A small number (105, 10.5\%) of respondents always chose the same alternative (A or B) for every choice set. Just over half $(596,59.5 \%)$ gave a consistent response to the repeated choice task.

\section{DCE Analysis}

We excluded 105 respondents who always chose the same alternative for every choice set. Therefore, the DCE analyses were based on 9 choices from each of 896 respondents, giving 8064 choice observations in total.

The MNL model results are presented in Table 3. For the unrestricted model, all symptom levels significantly impacted choice and in the expected direction with the exception of Mobility. For Mobility, there was one preference reversal (MOB2 and MOB3) which occurred in all models. Therefore, we estimated a "restricted" model where the coefficient for MOB2 and MOB3 was fixed to be the same. This resolved the preference reversal and did not impact model fit; the pseudo R2 was 0.164 for both the unrestricted and restricted models compared to a constants-only model.

\section{Rescaling on QALY Scale}

We used the restricted model to estimate utility weights for health states associated with AADC deficiency. It should be noted that incongruent observations (where the mean utility for the bedridden health state $>$ mean utility for the walking health state) were observed for $27 \%$ of respondents on the TTO task and $34 \%$ on the SG task. The following mean utility values represent the results with these responses removed from the respective tasks (the DCE values remained unchanged). From the vignette study, the estimated TTO utility weights for the best and worst health states were 0.5577 and 0.3891, respectively, ${ }^{19}$ for the SG task, 0.7093 and 0.5534 . The mean utility value for "bedridden" health state as rated on the HUI3 was 0.5263 (TTO) and 0.4924 (SG), and 0.5322 for the whole sample.

Table 4 shows some example health states and their corresponding utility values for the DCE health states after rescaling (the utility values derived using the SG and HUI3 are provided in Supplementary Tables 1 and $\underline{2}$, respectively). The difference in utility value between the best and worst health states was 0.169 (TTO). The largest relative disutility (ie, with the other attributes at the lowest, ie, "best" level) is associated with the "Mobility" attribute moving from "No problems walking" to "Bedridden": -0.0533 . The next largest disutility was also associated with "Mobility" moving from "Head control" to "Bedridden": -0.0305. For 
Table 2 DCE Participant Characteristics $(n=100 \mathrm{I})$

\begin{tabular}{|c|c|c|}
\hline Demographics & Gender, $\mathbf{N}(\%)$ & Age (SD) \\
\hline Female & $510(50.9)$ & $42.6(16.52)$ \\
\hline Male & $491(49.1)$ & 48.9 (16.47) \\
\hline \multicolumn{2}{|c|}{ Age group (N (\%)) } & Total \\
\hline \multicolumn{2}{|l|}{$18-34$} & $304(30.36)$ \\
\hline \multicolumn{2}{|l|}{$35-54$} & $333(33.26)$ \\
\hline \multicolumn{2}{|l|}{$55+$} & $364(36.36)$ \\
\hline \multicolumn{2}{|l|}{ Total } & $1001(100)$ \\
\hline \multicolumn{3}{|l|}{ Region (N (\%)) } \\
\hline \multicolumn{2}{|c|}{ Auvergne-Rhone-Alpes } & $125(12.48)$ \\
\hline \multicolumn{2}{|l|}{ Ile-de-France } & $189(18.88)$ \\
\hline \multicolumn{2}{|c|}{ Bourgogne-Franche-Comte } & $43(4.29)$ \\
\hline \multicolumn{2}{|l|}{ Bretagne } & $55(5.49)$ \\
\hline \multicolumn{2}{|c|}{ Centre-Val de Loire } & 39 (3.89) \\
\hline \multicolumn{2}{|l|}{ Corse } & $4(0.39)$ \\
\hline \multicolumn{2}{|l|}{ Grand Est } & $85(8.49)$ \\
\hline \multicolumn{2}{|l|}{ Hauts-de-France } & $92(9.19)$ \\
\hline \multicolumn{2}{|l|}{ Normandie } & 51 (5.09) \\
\hline \multicolumn{2}{|l|}{ Nouvelle-Aquitaine } & $92(9.19)$ \\
\hline \multicolumn{2}{|l|}{ Occitanie } & 91 (9.09) \\
\hline \multicolumn{2}{|l|}{ Pays de la Loire } & $59(5.89)$ \\
\hline \multicolumn{2}{|c|}{ Provence-Alpes-Cote d'Azur } & $74(7.39)$ \\
\hline \multicolumn{2}{|l|}{ Missing } & $2(0.19)$ \\
\hline \multicolumn{3}{|c|}{ Education (N (\%)) } \\
\hline \multicolumn{2}{|c|}{ Baccalaureat/brevet professionnel/equivalent } & $195(19.48)$ \\
\hline \multicolumn{2}{|l|}{ Brevet des colleges } & $274(27.37)$ \\
\hline \multicolumn{2}{|c|}{ CAP, BEP or Equivalent } & $225(22.47)$ \\
\hline \multicolumn{2}{|c|}{ Superieur court (niveau bac +2 ) } & $115(11.48)$ \\
\hline \multicolumn{2}{|c|}{ Superieur long (superieur bac +2 ) } & $185(18.48)$ \\
\hline \multicolumn{2}{|l|}{ Missing } & $7(0.69)$ \\
\hline
\end{tabular}

"Cognitive impairment" the disutility moving from the best to the worst health state for this attribute was -0.0284 . A similar disutility was also observed for the "Screaming" attribute when moving from the best to the worst health state: -0.0248 .
The disutilities of moving from the best and worst symptom levels for the other attributes were as follows: "Muscle weakness" -0.0234; "Feeding" -0.022 ; and "OGC" - 0.0167. In general, with the exception of the "Mobility" and "Cognitive Impairment" attributes, the differences between adjacent levels were small for the attributes. For instance, disutilities between adjacent health states ranged from -0.0091 between "No OGC" and "Occasional OGC" levels to -0.0153 between "Frequently screaming" and "Constantly screaming".

\section{Discussion}

The aim of this study was to generate utility values for AADC deficiency attributes using a discrete choice experiment in a representative French sample. The results demonstrated that the DCE parameters decreased or became more negative as attribute severity levels increased with the associated disutilities following the same pattern. The "Mobility" attribute appeared to be the main driver for the disutilities with "Head control", in particular, being associated with the greatest disutility.

These results contrast with those of the accompanying DCE undertaken with a UK sample where "Screaming" was the main driver followed by mobility. ${ }^{20}$ It was thought that in the UK study participants may have included parental or caregiver health-related quality of life (HRQoL) as part of their evaluation, rather than the child's HRQoL alone. This was supported by a low rating for parental and caregiver HRQoL in the corresponding vignette study. $^{11}$ Interviews with both parents of children with AADC deficiency and physicians treating these parents highlighted the importance of eye contact ${ }^{16}$ in the parent-child relationship. Head control would enable or at least facilitate the maintenance of eye contact. It may therefore be speculated that this could be the underlying reason for the relative importance attached to the "Mobility" attribute. This point is also further underlined by the fact that the associated French vignette study demonstrated consistently lower mean utility values for each health state for parents compared to those participants who were not parents. ${ }^{19}$ For instance, the range health state utilities for participants who were parents was 0.3402 to 0.4913 compared to 0.4141 to 0.5918 for nonparents. These results suggest that parental status has an impact on utility rating, although it must be noted that these differences were only observed on the TTO and not the SG task (for the current, French study). 
Table 3 DCE Data MNL Model Output

\begin{tabular}{|c|c|c|c|c|c|c|c|c|c|}
\hline & \multicolumn{4}{|c|}{ Unrestricted Model } & \multicolumn{5}{|c|}{ Restricted Model } \\
\hline & Coefficient & $P$ value & 95\% CI Lower & 95\% Cl Upper & & Coefficient & Prob $|z|>Z$ & 95\% Cl Lower & 95\% Cl Upper \\
\hline Constant & 0.09866 & $<0.0001$ & 0.04942 & 0.14789 & Constant & 0.09885 & 0.0001 & 0.04962 & 0.14808 \\
\hline ВМОВI & -0.27199 & $<0.0001$ & -0.39032 & -0.15366 & BMOBI & -0.27009 & $<0.0001$ & -0.3883 & -0.15188 \\
\hline BMOB2 & -0.39606 & $<0.0001$ & -0.51929 & -0.27284 & & & & & \\
\hline BMOB3 & -0.32682 & $<0.0001$ & -0.44288 & -0.21076 & ВMOB23 & -0.35713 & $<0.0001$ & -0.46114 & -0.25313 \\
\hline BMOB4 & -0.64118 & $<0.0001$ & -0.75617 & -0.52618 & BMOB4 & -0.63767 & $<0.0001$ & -0.75246 & -0.52288 \\
\hline BMOB5 & -1.49523 & $<0.0001$ & -1.62333 & -1.36713 & BMOB5 & -1.49244 & $<0.0001$ & $-1.6204 \mid$ & -1.36447 \\
\hline BWEAKI & -0.30862 & $<0.0001$ & -0.40317 & -0.21406 & BWEAKI & -0.3124 & $<0.0001$ & -0.40692 & -0.21788 \\
\hline BWEAK2 & -0.65234 & $<0.0001$ & -0.74754 & -0.55714 & BWEAK2 & -0.65385 & $<0.0001$ & -0.74901 & -0.55869 \\
\hline BOCGI & -0.20938 & $<0.0001$ & -0.2947 & -0.12407 & BOCGI & -0.21198 & $<0.0001$ & -0.29729 & -0.12667 \\
\hline BOCG2 & -0.4619 & $<0.0001$ & -0.54906 & -0.37474 & BOCG2 & -0.46646 & $<0.0001$ & -0.55331 & -0.37961 \\
\hline BFEEDI & -0.61412 & $<0.0001$ & -0.68476 & -0.54348 & BFEEDI & -0.61668 & $<0.0001$ & -0.68727 & -0.54609 \\
\hline BCOGI & -0.15271 & 0.0009 & -0.24253 & -0.06289 & BCOGI & -0.15513 & 0.0007 & -0.24483 & -0.06543 \\
\hline BCOG2 & -0.79856 & $<0.0001$ & -0.89099 & -0.70613 & BCOG2 & -0.79336 & $<0.0001$ & -0.88547 & -0.70126 \\
\hline BSCRMI & -0.2709 & $<0.0001$ & -0.36634 & -0.17545 & BSCRMI & -0.26725 & $<0.0001$ & -0.36249 & -0.17201 \\
\hline BSCRM2 & -0.70104 & $<0.0001$ & -0.80676 & -0.59533 & BSCRM2 & -0.69488 & $<0.0001$ & -0.80011 & -0.58964 \\
\hline
\end{tabular}


Table 4 DCE Health States and Utility Examples (TTO Anchoring)

\begin{tabular}{|c|c|c|c|c|c|c|}
\hline Mobility & Weakness & OGC & Feeding & Cognition & Screaming & U (HS) \\
\hline 1 & I & I & 1 & I & 1 & 0.5577 \\
\hline I & 1 & I & 1 & 2 & I & 0.5522 \\
\hline I & 1 & 2 & 1 & 1 & I & $0.550 \mathrm{I}$ \\
\hline I & I & I & 1 & 1 & 2 & $0.548 \mathrm{I}$ \\
\hline 2 & I & I & 1 & I & 1 & 0.5480 \\
\hline I & 2 & I & 1 & 1 & I & 0.5465 \\
\hline 4 & 1 & I & 1 & 1 & 1 & 0.5449 \\
\hline 3 & 1 & I & 1 & 1 & 1 & 0.5449 \\
\hline I & 1 & 3 & 1 & 1 & 1 & 0.5410 \\
\hline I & 1 & I & 2 & 1 & I & 0.5357 \\
\hline 5 & I & I & 1 & I & I & 0.5349 \\
\hline I & 3 & I & 1 & I & I & 0.5343 \\
\hline I & 1 & I & 1 & 1 & 3 & 0.5329 \\
\hline I & 1 & I & 1 & 3 & I & 0.5293 \\
\hline 3 & 3 & I & 1 & 1 & 1 & 0.5216 \\
\hline 4 & 1 & I & 1 & 1 & 3 & $0.520 \mathrm{I}$ \\
\hline I & 1 & 3 & 1 & 1 & 3 & 0.5162 \\
\hline 4 & 1 & 2 & 3 & 1 & 3 & 0.5125 \\
\hline 6 & 1 & I & 1 & 1 & I & 0.5044 \\
\hline 5 & 2 & 2 & 3 & 2 & 2 & 0.5011 \\
\hline 2 & 2 & 2 & 2 & 2 & 2 & 0.4922 \\
\hline 3 & 2 & 2 & 2 & 2 & 2 & $0.489 \mid$ \\
\hline 6 & 1 & 2 & 3 & I & 2 & 0.4872 \\
\hline 3 & 1 & 2 & 2 & 3 & I & 0.4870 \\
\hline 5 & 2 & I & 1 & 3 & 2 & 0.4858 \\
\hline 5 & 2 & 3 & 3 & 3 & I & 0.4787 \\
\hline 3 & 2 & 2 & 2 & 2 & 3 & 0.4738 \\
\hline I & 3 & 2 & 1 & 3 & 3 & 0.4736 \\
\hline 3 & 3 & I & 2 & 3 & 2 & 0.4616 \\
\hline 6 & 3 & 3 & 2 & 3 & 3 & $0.389 \mid$ \\
\hline
\end{tabular}

Abbreviation: OGC, oculogyric crises.

One other set of contrasts to be noted between the current, French study and its UK counterpart ${ }^{11}$ is both the difference between the health states as well as the level of each health state. Participants in the UK study rated the health states, on average, higher than their French counterparts, ie, 0.494 and 0.7279 for the "Bedridden" and 
"Walking with assistance" health states, respectively, compared to 0.3891 to 0.5577 for the French sample. The difference between these was also smaller for the French sample, ie, 0.1686 compared to 0.2339 for the UK. It is evident that the French participants were rating the health state, and consequently by inference the symptoms and impact of AADC deficiency more severely than the UK participants. The "best" health state ("Walking with assistance"), in particular, was rated much lower by the French participants. There may be a number of reasons underlying these differences, including cultural variation, for instance relative preferences for "head control" (France) over "screaming" (UK), however it may also be speculated that sociodemographics could have played a hand here: the number of participants who were parents was lower in the UK sample, ie, $26.6 \%$ compared to $35 \%$. Given the differences noted above, it may be that parental status also impacted on the divergence observed at a country level.

One potential limitation resulting from the narrow range of health state utilities observed in the associated (French) vignettes study ${ }^{20}$ was that the disutilities separating adjacent health states, especially those with less severe symptomatology were relatively small. As a consequence of which given these utilities were used to anchor the DCE parameters - the disutilities for individual symptoms observed in this study were also small. The lower anchor employed in this study (derived from the TTO vignettes ${ }^{19}$ ) did not allow any health states to be considered worse than dead (WTD). Some studies in rare, life-limiting conditions ${ }^{7}$ have allowed health states to be rated as WTD (in proxy ratings, rather than, for instance TTO or SG tasks).

The mean health state utility used as the anchor in the current study was 0.3891 . Furthermore, around 35\% of participants rated the worst ("Bedridden") health state at zero, or "death". These results taken together suggest that the majority of participants did not classify the worst health state as being severe enough to be WTD. However, it is, of course, feasible that should participants have been able to rate vignettes WTD, that the DCE estimates would have extended to a larger range.

Additionally, the $\mathrm{HUI}^{22,23}$ had been included as a potential anchor for the "Bedridden" health state in the current study. This instrument had been selected ahead of other preference-based measures, such as the EQ-5D, ${ }^{3}$ as it includes domains more relevant to AADC deficiency, for instance, Vision, Speech and Dexterity. However, the mean health state utility on the HUI3 was relatively high at 0.5263 (for the "Bedridden" health state) meaning that there would have been little, if any, differentiation between the "worst" and "best" health states were the mean HUI3 utility value to be applied as the anchor for the recalibration.

The mean HUI3 level may reflect a lack of understanding or comprehension on the part of participants and may be indicative, in general, of the problems inherent in DCE tasks where individuals (drawn from the general population) are faced with rare conditions and symptoms such as, for instance, oculogyric crises (OCG), which are most likely to be unfamiliar to those participants. Nevertheless, it should also be stressed that these symptoms of AADC deficiency were derived from extensive content validation involving parents and caregivers of, as well as physicians treating AADC deficiency patients. ${ }^{16}$ The use of vignettes may have also contributed to this; however, given the rarity of AADC deficiency and the nature of the patients affected (predominantly very young children), this is the only methodology available ${ }^{27}$ to generate health state utilities in this population.

\section{Conclusion}

This is the first study, to our knowledge, to derive robust health utilities for AADC deficiency symptoms using a large sample drawn from the general French population and to some extent contrast to the findings from a UK-based study to derive utilities using similar methods. These data will be utilised to enable improvements in health-related quality of life to be considered in a country-specific economic evaluation of a gene therapy for AADC deficiency.

\section{Acknowledgments}

This work was supported by PTC Therapeutics ("PTC"). An abstract of this paper was presented at the Virtual Conference of the International Society for Pharmacoeconomics and Outcomes Research (ISPOR) - North America 2021 (May 17-20) as a poster presentation.

\section{Disclosure}

KB and IBOZ are employees of PTC Therapeutics. ABS and $\mathrm{AH}$ are employees of York Health Economics Consortium (YHEC). YHEC was commissioned by PTC to undertake the study. JAW received a consulting fee via her employer (University of East Anglia) from YHEC for her contribution to the study. FH is a consultant at AEC Partners. She worked full time for PTC Therapeutics during the study design and during the study. She worked on an as-needed basis during 
the manuscript preparation. She also reports that AEC Partners received fees from PTC Therapeutics during this time. GP reports personal fees from PTC Therapeutics for design of the study, support for the collection of data in France, and revision of the manuscript. The authors report no other conflicts of interest in this work.

\section{References}

1. Kojima K, Nakajima T, Taga N, et al. Gene therapy improves motor and mental function of aromatic 1-amino acid decarboxylase deficiency. Brain. 2019;142(2):322-333. doi:10.1093/brain/awy331

2. Wassenberg T, Molero-Luis M, Jeltsch K, et al. Consensus guideline for the diagnosis and treatment of aromatic 1-amino acid decarboxylase (AADC) deficiency. Orphanet J Rare Dis. 2017;12(1):12.

3. Dolan P. Modeling valuations for EurQol health states. Med Care. 1997;35:1095-1108. doi:10.1097/00005650-199711000-00002

4. Haute Autorité de Santé (HAS). Choices in methods for economic evaluation; 2020.

5. Landfeldt E, Edström J, Sejersen T, Tulinius M, Lochmüller H, Kirschner J. Quality of life of patients with spinal muscular atrophy: a systematic review. Eur J Paediatr Neurol. 2019;23(3):347-356. doi:10.1016/j.ejpn.2019.03.004

6. Landfeldt E, Lindgren P, Bell CF, et al. Health-related quality of life in patients with Duchenne muscular dystrophy: a multinational, cross-sectional study. Dev Med Child Neurol. 2016;58(5):508-515. doi:10.1111/dmcn.12938

7. Lloyd AJ, Thompson R, Gallop K, Teynor M. Estimation of the quality of life benefits associated with treatment for spinal muscular atrophy. Clinicoecon Outcomes Res. 2019;11:615-622. doi:10.2147/ CEOR.S214084

8. Pickard AS, Knight SJ. Proxy evaluation of health-related quality of life: a conceptual framework for understanding multiple proxy perspectives. Med Care. 2005;43(5):493-499. doi:10.1097/01. mlr.0000160419.27642.a8

9. Torrance GW. Measurement of health state utilities for economic appraisal. J Health Econ. 1986;5:1-30. doi:10.1016/0167-6296(86) 90020-2

10. Smith AB, Retzler J, Taylor MJ. Standard gamble to derive utility health states for limbal stem cell deficiency. Clinicoecon Outcomes Res. 2020;535:546.

11. Smith AB, Hanbury A, Buesch K. Eliciting health state utilities for aromatic L-amino acid decarboxylase (AADC) deficiency: a UK vignette study. J Patient Rep Outcomes. 2021;5(1). doi:10.1186/ s41687-021-00403-0

12. Ryan M, Farrar S. Eliciting preference for healthcare using conjoint analysis. BMJ. 2000;320:1530-1533. doi:10.1136/bmj.320.7 248.1530

13. Toumi M, Millier A, Cristeau O, Thokagevistk-Desroziers K, Dorey J, Aballéa S. Social preferences for orphan drugs: a discrete choice experiment among the French general population. Front Med. 2020;7:323. doi:10.3389/fmed.2020.00323

Patient Related Outcome Measures

\section{Publish your work in this journal}

Patient Related Outcome Measures is an international, peer-reviewed, open access journal focusing on treatment outcomes specifically relevant to patients. All aspects of patient care are addressed within the journal and practitioners from all disciplines are invited to submit their work as well as healthcare researchers and patient support groups.
14. López-Bastida J, Ramos-Goñi JM, Aranda-Reneo I, Taruscio D, Magrelli A, Kanavos P. Using a stated preference discrete choice experiment to assess societal value from the perspective of patients with rare diseases in Italy. Orphanet $J$ Rare Dis. 2019;14(1):154. doi:10.1186/s13023-019-1126-1

15. Gu NY, Botteman MF, Gerber RA, et al. Eliciting health state utilities for Dupuytren's contracture using a discrete choice experiment. Acta Orthop. 2013;84(6):571-578. doi:10.3109/17453674.2013.865097

16. Hanbury A, Smith AB, Buesch K. Deriving vignettes for the rare disease AADC using parent, caregiver and clinician interviews to evaluate the impact on health-related quality of life. Patient Relat Outcome Meas. 2021;12:1-12.

17. Smith AB, Hanbury A, Ortiz de Zarate IB, Hammes F, de Pourvourville G, Buesch K. Eliciting health state utilities for aromatic L-amino acid decarboxylase (AADC) deficiency: a vignette study in France. Patient Relat Outcome Meas. 2021;12:237-246. doi:10.2147/PROM.S306228

18. Smith AB, Hanbury A, Whitty JA, Buesch K. A discrete choice experiment to derive health utilities for aromatic L-amino acid decarboxylase (AADC) deficiency. Patient Relat Outcome Meas. 2021;12:97-106. doi:10.2147/PROM.S294628

19. Marshall D, Bridges JFP, Hauber B, et al. Conjoint analysis applications in health - how are studies being designed and reported? Patient. 2010;3(4):249-256. doi:10.2165/11539650-00000000000000

20. Orme BK. Getting Started with Conjoint Analysis: Strategies for Product Design and Pricing Research. Madison Wisconsin: Research Publishers LLC; 2006.

21. Rose JM, Collins AT, Bliemer MCJ, Hensher DA. Ngene [Computer Program]. Version 1.1.1 ed. ChoiceMetrics Pty Ltd; 2012.

22. Feeny D, Furlong W, Boyle M, Torrance GW. Multi-attribute health status classification systems: health utilities index. Pharmacoeconom. 1995;7(6):490-502. doi:10.2165/00019053-199507060-00004

23. Feeny DH, Furlong WJ, Torrance GW, et al. Multi-attribute and single-attribute utility functions for the health utilities index mark 3 system. Med Care. 2002;40:113-128. doi:10.1097/00005650200202000-00006

24. Costet N, Le Galès C, Buron C, et al. French cross-cultural adaptation of the health utilities indexes mark 2 (HUI2) and 3 (HUI3) classification systems. Clinical and Economic Working Groups. Qual Life Res. 1998;7:245-256. doi:10.1023/A:1008830115246

25. Le Galès C, Buron C, Costet N, Rosman S, Slama G. Development of a preference-weighted health status classification system in France: the health utilities index 3. Health Care Manag Sci. 2002;5:41-51.

26. Econometric Software Inc. NLOGIT [Computer program]. Version 5 ed. Plainview, NY: Econometric Software, Inc.; 2012.

27. Matza LS, Stewart KD, Lloyd AJ, Rowen D, Brazier JE. Vignettebased utilities: usefulness, limitations, and methodological recommendations. Value Health. 2021;24:812-821. doi:10.1016/j. jval.2020.12.017

The manuscript management system is completely online and includes a very quick and fair peer-review system. Visit http://www. dovepress.com/testimonials.php to read real quotes from published authors. 